\title{
Condrosarcoma del hueso hioides: a propósito de un caso
}

\section{Chondrosarcoma of the hyoid bone: a case report}

\author{
Andrés González F. ${ }^{1}$ Ignacio Sanhueza T. ${ }^{1}$, Diego Regalado B. ${ }^{2}$
}

'Servicio de Otorrinolaringología, Complejo Hospitalario De Navarra. Pamplona, España.

${ }^{2}$ Servicio de Otorrinolaringología, Hospital Reina Sofía. Tudela, España.

Los autores declaran no tener conflictos de interés.

Recibido el 28 de septiembre de 2020. Aceptado el 17 de enero de 2021.

Correspondencia: Andrés González F.

Calle Carmen $11,1^{\circ}$ A Pamplona

(31001). Navarra, España. Email: andresgonfer@hotmail.com

\begin{abstract}
Resumen
Los condrosarcomas son cánceres realmente infrecuentes en cabeza y cuello, y más aún en el hueso hioides. Por lo general, son neoplasias que debutan como una masa cervical sin otra sintomatología. Su diagnóstico requiere de estudios de imagen y su tratamiento es fundamentalmente quirúrgico. Comentamos el caso de un paciente de 57 años, desde el diagnóstico de la lesión hasta su tratamiento y seguimiento, y una revisión bibliográfica de esta patología.

Palabras clave: Condrosarcoma, hioides, cabeza, cuello, sarcoma.
\end{abstract}

\begin{abstract}
Chondrosarcomas are a rare type of head and neck cancer, especially in the hyoid bone. They usually make their debut through a cervical mass, without other symptoms. The diagnosis requires image studies, and the treatment is fundamentally surgical. We report the case of a 57 years old patient, from the lesion diagnosis, its treatment and follow up, and a bibliographic review of this pathology.

Keywords: Chondrosarcoma, hyoid, head, neck, sarcoma.
\end{abstract}

\section{Introducción}

Los condrosarcomas son muy poco frecuentes en cabeza y cuello; y aún son más inusuales aquellos que asientan en el hueso hioides. Debutan como una masa indolora en la región central del cuello y requieren de estudios de imagen para su correcto diagnóstico. El tratamiento de elección es la exéresis quirúrgica con márgenes de seguridad, pudiendo ofrecerse radioterapia complementaria en muy seleccionados casos. El pronóstico de esta patología oncológica depende en gran medida de su índice de malignidad, a su vez dependiente de sus características histopatológicas. De modo que la supervivencia a largo plazo decrece cuanto mayor es el grado de malignidad de esta lesión.

\section{Caso Clínico}

Describimos el caso de un paciente de 57 años que, tras un intento de suicidio por ahorcamiento y posterior estudio de imagen con una tomografía computarizada (TC) cervical para valoración de las posibles lesiones producidas, se identifica una lesión osteolítica de $3,5 \mathrm{~cm}$ de diámetro en espacio parafaríngeo derecho con calcificaciones dispersas en su interior (Figura 1). El informe radiológico es compatible con un condrosarcoma.

En la exploración nasofibrolaringoscópica del paciente se evidencia una masa en el espacio parafaríngeo derecho que desplaza la laringe hacia el lado contralateral. Tras ser valorado por el comité oncológico multidisciplinario de cabeza y cuello, se decide realizar estudio de resonancia magnética cervical (RM) para descartar compromiso vascular que imposibilite la intervención. Tras esto, se decide exéresis quirúrgica de la lesión (Figura 2), mediante una incisión centrocervical, inmediatamente superficial al hueso hioides, disección por planos, y exéresis de lesión y hemicuerpo derecho de hioides. No se envían muestras intraoperatorias. No se lleva a cabo linfadenectomía cervical. La lesión se 


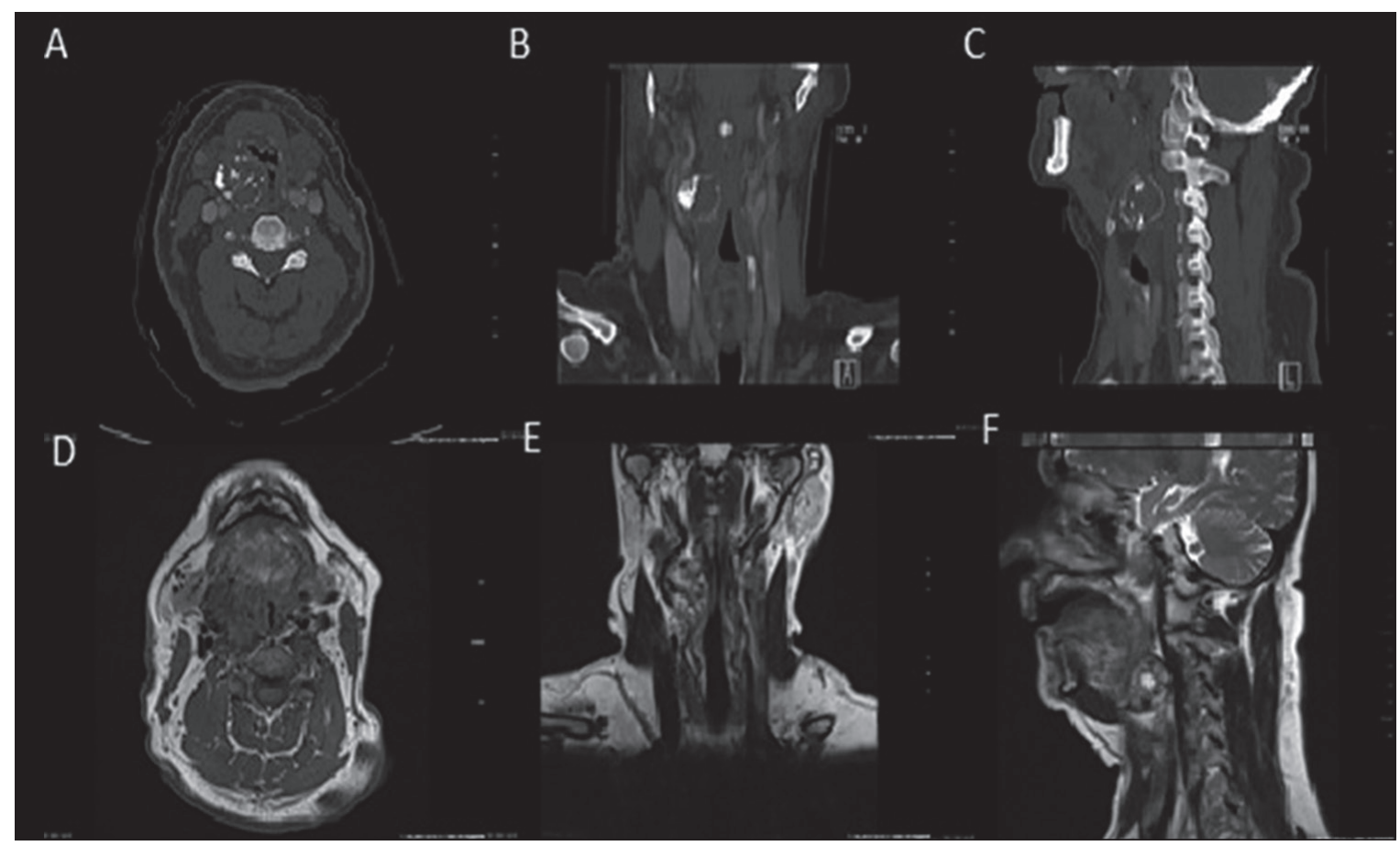

Figura 1. Imágenes del caso clínico. A, B y C) Cortes axial, coronal y sagital de lesión en TC. D, E y F) Cortes axial, coronal y sagital de lesión en resonancia magnética.

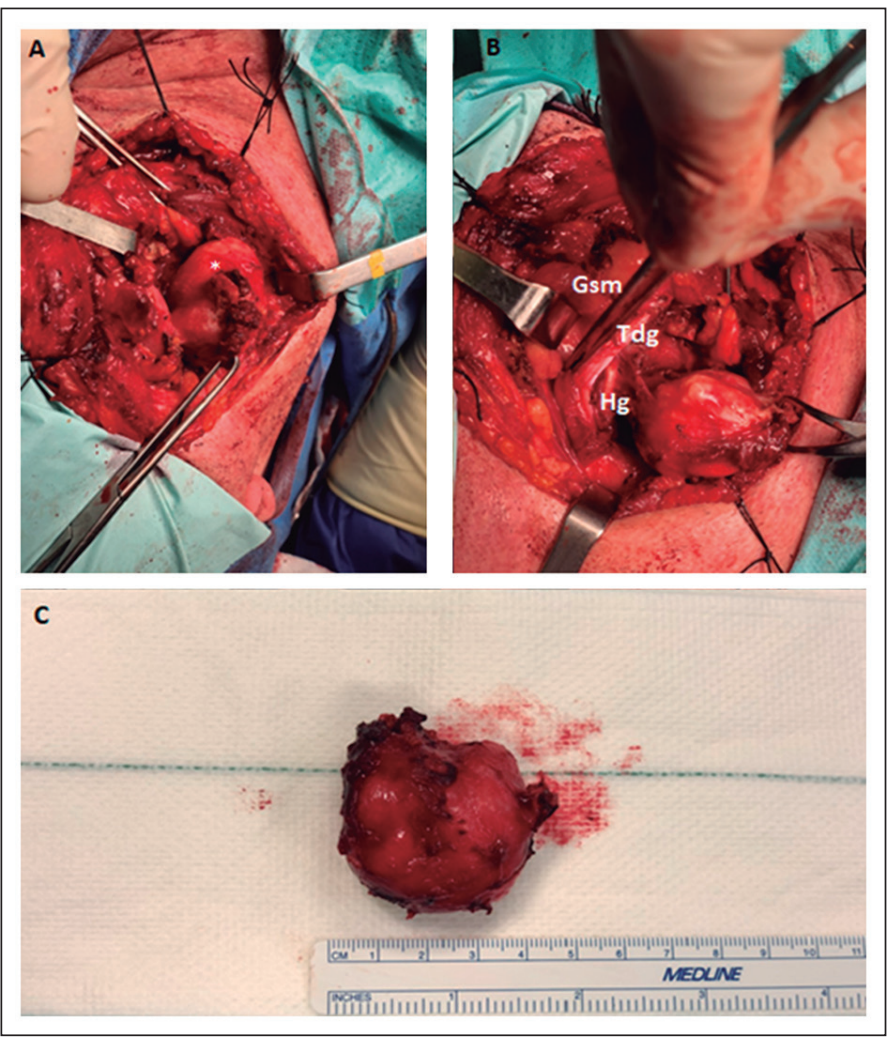

Figura 2. A) Esqueletización del hueso hioides de su musculatura superior e inferior, observando masa redondeada en asta mayor derecha. B) Exéresis completa de la lesión. En la imagen se observa la glándula submaxilar derecha (Gsm), tendón del digástrico (Tdg) y nervio hipogloso derecho $(\mathrm{Hg})$. C) Pieza quirúrgica completa, de aproximadamente $3,5 \mathrm{~cm}$ de diámetro mayor. 
encuentra encapsulada por el periostio. No se realizó estudio anatomopatológico previo a la cirugía.

\section{Discusión}

Los condrosarcomas son cánceres óseos caracterizados por la formación de matriz cartilaginosa $^{1,2}$. Suponen el $11 \%$ del total de cánceres óseos primarios y típicamente asientan en pelvis, costillas y huesos largos ${ }^{1,2}$. Su aparición en cabeza y cuello es de tan solo el 1\%-12\% de los casos $^{1}$, siendo, en estas localizaciones, más frecuente en la base del cráneo, cavidad nasal, maxilar, mandíbula y esqueleto laríngeo ${ }^{2,3}$. La aparición de un condrosarcoma en el hueso hioides es extremadamente rara aunque deben tenerse en cuenta como diagnóstico diferencial de una masa submandibular4.

Este tipo de tumores se pueden dividir en primarios o secundarios. Los primarios son inusuales y suelen diagnosticarse en la infancia. Los secundarios se originan de lesiones preexistentes como osteocondromas o encondromas y suelen verse entre los 30-60 años ${ }^{2}$. En este artículo se describe el caso de un varón de 57 años que debuta con esta lesión, sin evidencia de alguna previa.

Histológicamente, se clasifican en 3 grados de malignidad en relación con su índice mitótico, tamaño nuclear, patrón de tinción y celularidad. El condrosarcoma de grado 1 (bajo grado) es el más habitual y supone el 60\%-65\% de los casos. El de grado 2, alrededor del 30\%$35 \%$ y el de grado 3 , el 5\%-10\% restante ${ }^{5}$. Este último subgrupo es el que presenta mayor potencial metastático, siendo los pulmones y los huesos las regiones donde más frecuentemente disemina. El informe anatomopatológico de la pieza quirúrgica completa del paciente descrito es compatible con un condrosarcoma de grado 1 .

La presentación clínica más habitual suele ser una masa indolora en la región central del cuello $^{2,3,6}$, lo que se corresponde con el caso descrito. El TC de cuello es útil demostrando las calcificaciones características y la rotura de la cortical ósea. Sin embargo, la RM es más sensible, ya que los condrosarcomas típicamente son hipointensos con realce periférico en $\mathrm{T} 1$, e hiperintensos en $\mathrm{T} 2^{6}$. El diagnóstico histo- lógico puede hacerse con una punción con aguja fina o gruesa, no siendo recomendada la biopsia abierta de la lesión por el riesgo de diseminación ${ }^{5}$.

La cirugía suele ser el tratamiento de elección. Si la resección es completa, no hace falta un tratamiento adyuvante porque el tumor suele estar encapsulado en el periostio, sin invasión de tejidos vecinos. En todos los grados de condrosarcoma, hay un 5\%-7\% de posibilidad de afectación ganglionar, por lo que no se recomienda el vaciamiento cervical funcional ${ }^{6}$. Sin embargo, si los márgenes quirúrgicos no son adecuados, es recomendable ampliar la cirugía antes de ofrecer radioterapia ${ }^{6}$. En caso de ser necesaria la radioterapia, se recomiendan dosis $>70$ Gy para tumores no resecables, o de 60-70 Gy en caso de márgenes quirúrgicos afec$\operatorname{tos}^{5}$. La radioterapia también debe ofrecerse como tratamiento adyuvante en aquellos casos de alto grado de malignidad. Una revisión sistemática de 107 casos de condrosarcoma en hueso y tejidos blandos sugiere que hay un mejor control de la enfermedad en pacientes que recibieron radioterapia complementaria, aunque la diferencia no fue significativa ${ }^{7}$. En nuestro caso, la cirugía consiguió la exéresis completa, confirmando la ausencia de invasión de márgenes por el estudio anatomopatológico posquirúrgico, y al ser de bajo grado no recibió tratamiento adyuvante.

La terapia fotodinámica es anecdótica. Sólo está descrita en el caso de un paciente que rechazó la cirugía por las posibles secuelas funcionales de la misma ${ }^{8}$. Las posibles secuelas quirúrgicas incluyen disfonía y disfagia. La primera ocurre si hay afectación laríngea y la segunda se debe a la resección total o parcial del hueso del hioides". En el caso descrito, existe disfagia consecuente de la intervención, con clara mejoría progresiva mediante rehabilitación.

La recurrencia y las metástasis son habituales en zonas en donde no es posible asegurar un margen quirúrgico adecuado. Esto sucede en ciertas áreas de la cabeza y el cuello, costillas o pubis. La tasa de supervivencia es de $90 \%$ para el grado 1 , de $81 \%$ para el grado $2 \mathrm{y}$ del $43 \%$ para el grado 32 . Esto se debe a que las metástasis a distancia son raras en los de tipo 1 , pero pueden llegar hasta el $70 \%$ en los de tipo $3^{10}$. Esta es la razón por la que estos pacientes 
deben tener un seguimiento estrecho, que debe de incluir una placa de tórax cada 6 meses al ser el pulmón el sitio de metástasis más habitual $^{2}$. Nuestro paciente, después de 12 meses de seguimiento, no presenta recidiva.

\section{Conclusión}

El condrosarcoma es un tumor poco habitual dentro de la oncología de cabeza y cuello. Tanto el TC como la RM son útiles en el diagnóstico, pero es fundamental contar con la histología para el pronóstico. La resección quirúrgica es el tratamiento de elección. Es necesario un seguimiento del paciente a largo plazo por la posibilidad de recidiva.

\section{Bibliografía}

1. Tachino H, Fushiki H, Ishida M, Watanabe Y. A LowGrade Chondrosarcoma Presenting as an Unusual Cervical Mass in the Hyoid Bone: A Case Report. J Med Case Rep. 2012;6:21. doi: 10.1186/1752-1947-621.

2. Caglar O, Karatag O, Avci A, Derekoy S. An Unusual Cervical Mass in the Hyoid Bone: IntermediateGrade Chondrosarcoma. Case Rep Otolaryngol. 2019;2019:7170832 .doi: 10.1155/2019/7170832.

3. Horta M, Fernandes L, Borges A. Chondrosarcoma of the Hyoid Bone: An Atypical Site of a Sarcoma of the Head and the Neck.
BMJ Case Rep. 2015;2015:bcr2015212291. doi: 10.1136/bcr-2015-212291.

4. Rito M, Cabrera RA. Chondrosarcoma of the hyoid bone: A diagnostic pitfall in the submandibular region. Diagn Cytopathol. 2015;43(11):933-936. doi: $10.1002 /$ dc. 23328 .

5. Maki D, Mori T, Teshima M, et al. Chondrosarcoma of the hyoid bone - Report of a case and a literature review of the suitable treatment strategy. Auris Nasus Larynx. 2017;44(5):629-634. doi: 10.1016/j. anl.2016.09.004.

6. Zhang I, Zaorsky NG, Abraham JA, Tuluc M, Curry JM, Bar-Ad V. Chondrosarcoma of the hyoid bone: case report and review of current management options. Head Neck. 2014;36(7):E65-E72. doi: 10.1002/hed.23373.

7. Xu J, Li D, Xie L, Tang S, Guo W. Mesenchymal chondrosarcoma of bone and soft tissue: a systematic review of 107 patients in the past 20 years. PLoS One. 2015;10(4):e0122216. doi: 10.1371/journal. pone. 0122216.

8. Nhembe F, Jerjes W, Upile T, Hamdoon Z, Hopper C. Chondrosarcoma of the hyoid treated with interstitial photodynamic therapy: case study. Photodiagnosis Photodyn Ther. 2009;6(3-4):235-237. doi: 10.1016/j. pdpdt.2009.09.004.

9. Hirunpat S, Prueganusak K, Sinruk Y, Tanomkiat $\mathrm{W}$. Chondrosarcoma of the hyoid bone: imaging, surgical, and histopathologic correlation. AJNR Am J Neuroradiol. 2006;27(1):123-125.

10. Saki N, Akhlagh SN, Mostofi NE, Ahmadi K. Chondrosarcoma of the hyoid bone: imaging, surgical, and histopathologic correlation. Laryngoscope. 2008;118(7):1211-1213. doi: 10.1097/MLG.0b013e318170f8bd. 